\title{
Bowl-Like C@MoS 2 Nanocomposites as Anode Materials for Lithium-Ion Battery: Enhanced Stress Buffering and Charge/Mass Transfer
}

Xiue Zhang ${ }^{1,3}$, Xing Chen ${ }^{1}$, Huajuan Ren ${ }^{1}$, Guowang Diao ${ }^{1}$, Ming Chen ${ }^{1,2}$ *, Shaowei Chen ${ }^{* 3}$

${ }^{1}$ School of Chemistry and Chemical Engineering, Yangzhou University, 180 Siwang Ting Road, Yangzhou 225002, P. R. China

${ }^{2}$ Key Laboratory of Advanced Energy Materials Chemistry (Ministry of Education), College of Chemistry, Nankai University, 94 Weijin Road, Tianjin 300071, P. R. China

${ }^{3}$ Department of Chemistry and Biochemistry, University of California, 1156 High Street, Santa Cruz, California 95064, USA

Number of pages: 9

Number of tables: 2

\footnotetext{
* Corresponding Authors

E-mail address: chenming@yzu.edu.cn; shaowei@ucsc.edu
} 
Number of figures: 10
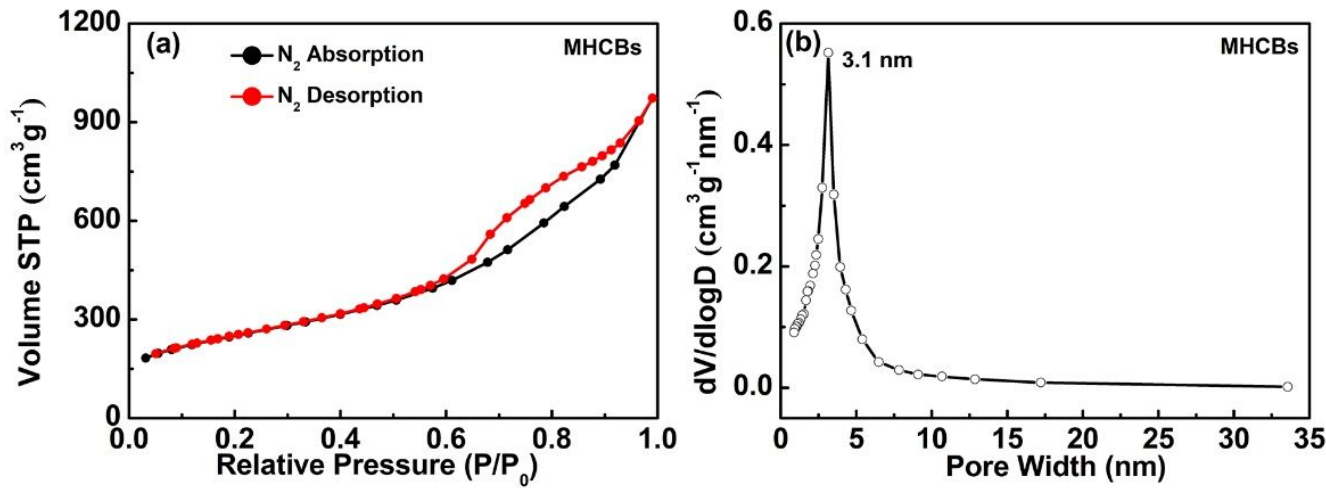

Figure S1. BET nitrogen adsorption and desorption isotherms (a) and pore size distribution of MHCBs (b).

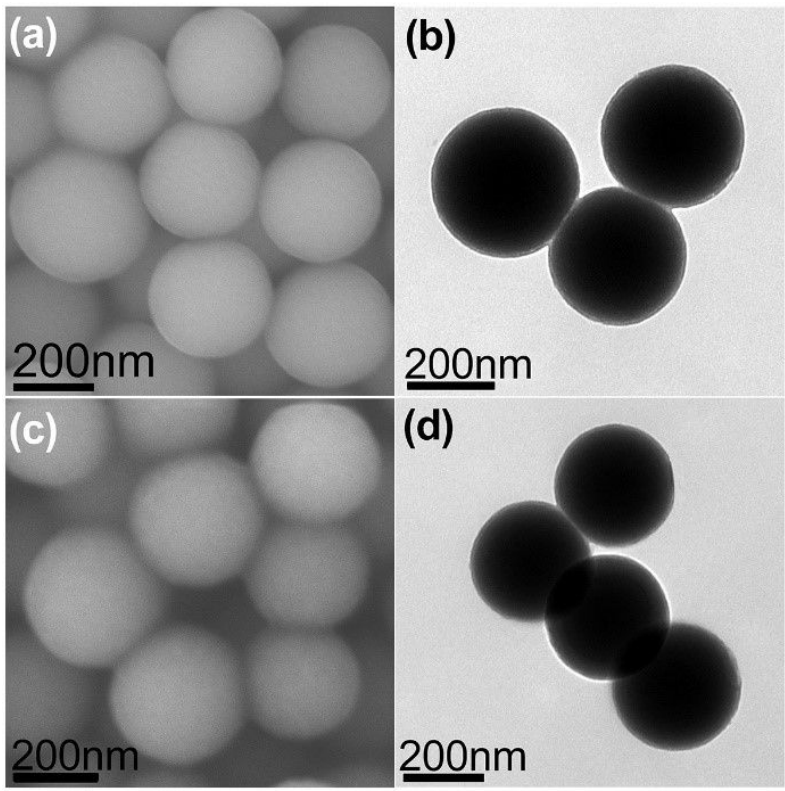

Figure S2. FESEM and TEM images of $(\mathrm{a}, \mathrm{b}) \mathrm{SiO}_{2} @ \mathrm{SiO}_{2} / \mathrm{RF}$ nanospheres and (c,d) $\mathrm{SiO}_{2} @ \mathrm{SiO}_{2} / \mathrm{C}$ nanospheres for the preparation of MHCBs. 


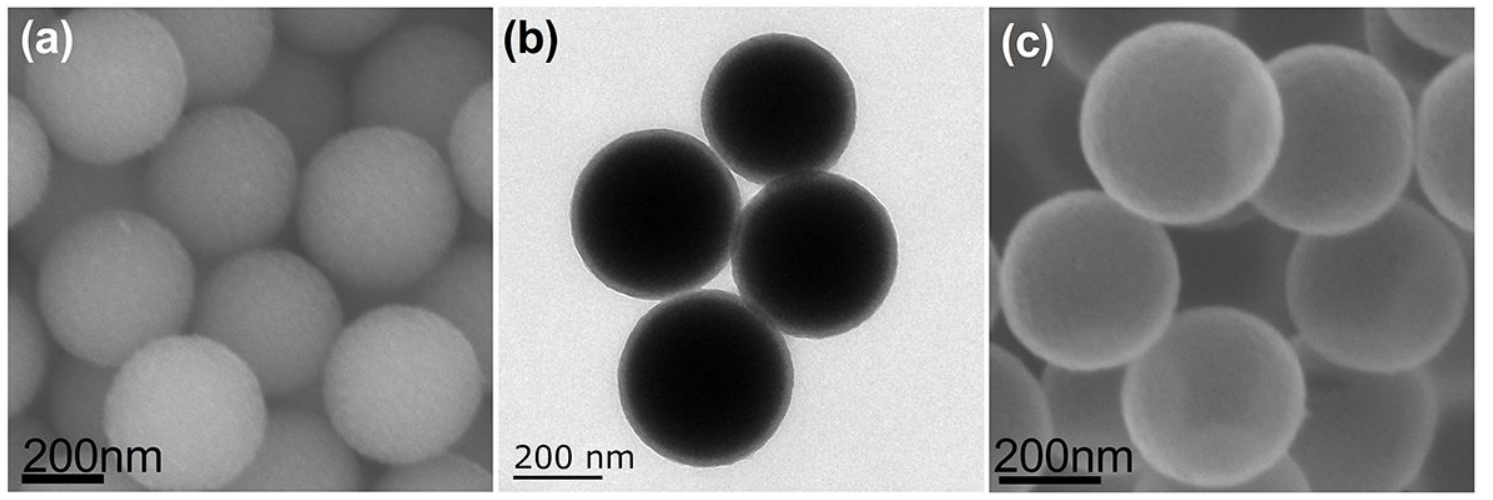

Figure S3. FESEM and TEM images of $(a, b) \mathrm{SiO}_{2} @ \mathrm{SiO}_{2} / \mathrm{C}$ nanospheres and (c) the resulting mesoporous hollow carbon spheres (MHCSs).
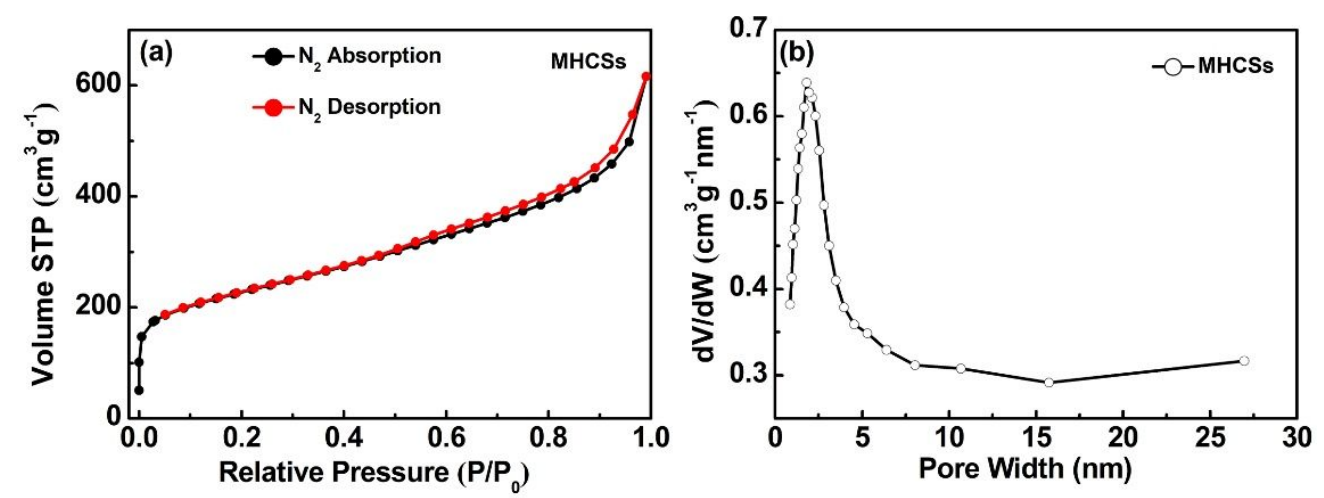

Figure S4. BET nitrogen adsorption and desorption isotherms (a) and pore size distribution of MHCSs (b). 


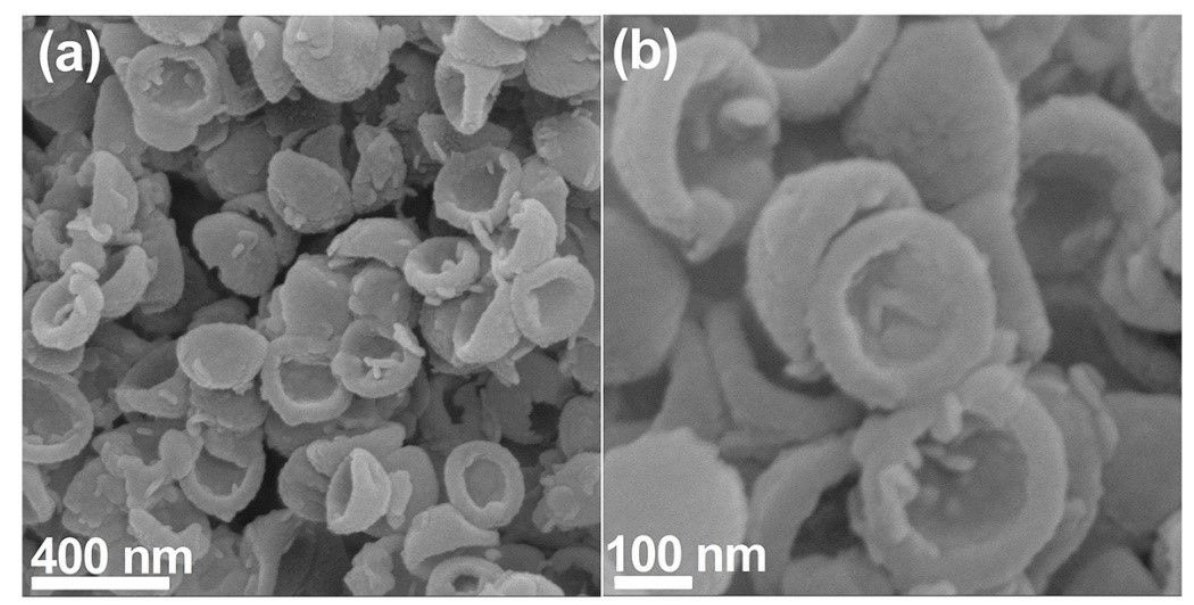

Figure S5. (a, b) SEM images of bowl-like $\mathrm{C} @ \mathrm{MoS}_{2}$ nanocomposites.

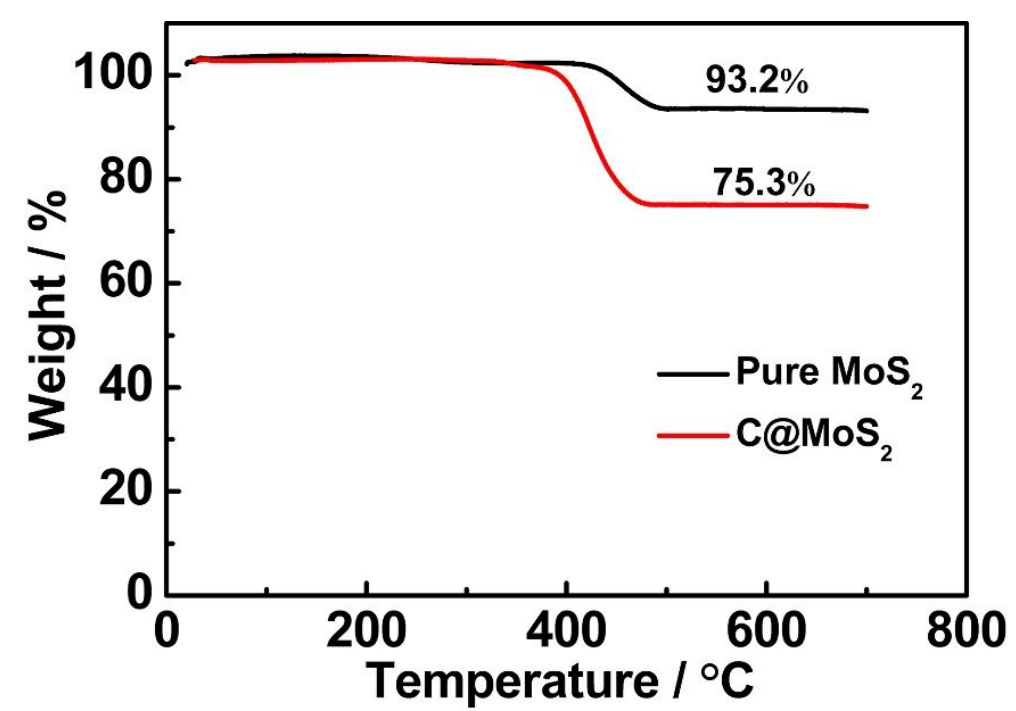

Figure S6. TGA curves of pure $\mathrm{MoS}_{2}$ and bowl-like $\mathrm{C} @ \mathrm{MoS}_{2}$.

Under air atmosphere, the pure $\mathrm{MoS}_{2}$ undergoes a weight loss owing to the oxidative reaction of $\mathrm{MoS}_{2}$ to $\mathrm{MoO}_{3}\left(2 \mathrm{MoS}_{2}+7 \mathrm{O}_{2} \rightarrow 2 \mathrm{MoO}_{3}+4 \mathrm{SO}_{2}\right)$. The weight loss is about $6.8 \%$. In other words, the mass ratio of $\mathrm{MoO}_{3} / \mathrm{MoS}_{2}$ is $93.2 \%$.

The weight fraction of $\mathrm{MoS}_{2}$ in bowl-like $\mathrm{C} @ \mathrm{MoS}_{2}$ is calculated as follows: Assuming the mass of $\mathrm{MoS}_{2}$ and carbon in the bowl-like $\mathrm{C} @ \mathrm{MoS}_{2}$ to be $\mathrm{x}, \mathrm{y}$, respectively, and the carbon is completely removed after combustion and $\mathrm{MoS}_{2}$ is transformed into $\mathrm{MoO}_{3}$, there is one relation between the final mass and initial mass, $0.932 \mathrm{x} /(\mathrm{x}+\mathrm{y})=0.753$. Therefore $\mathrm{x} /(\mathrm{x}+\mathrm{y})$ $=0.81$, the weight fraction of $\mathrm{MoS}_{2}$ in bowl-like $\mathrm{C} @ \mathrm{MoS}_{2}$ is $81 \mathrm{wt} \%$. 

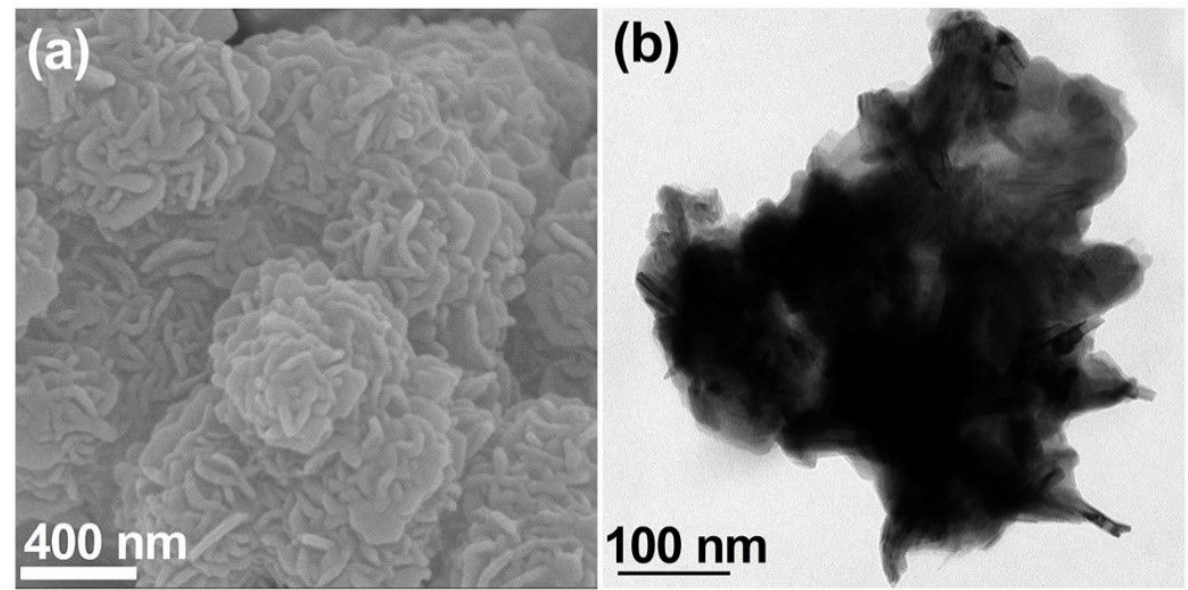

Figure S7. (a) SEM images and (b) TEM images of pure $\mathrm{MoS}_{2}$.
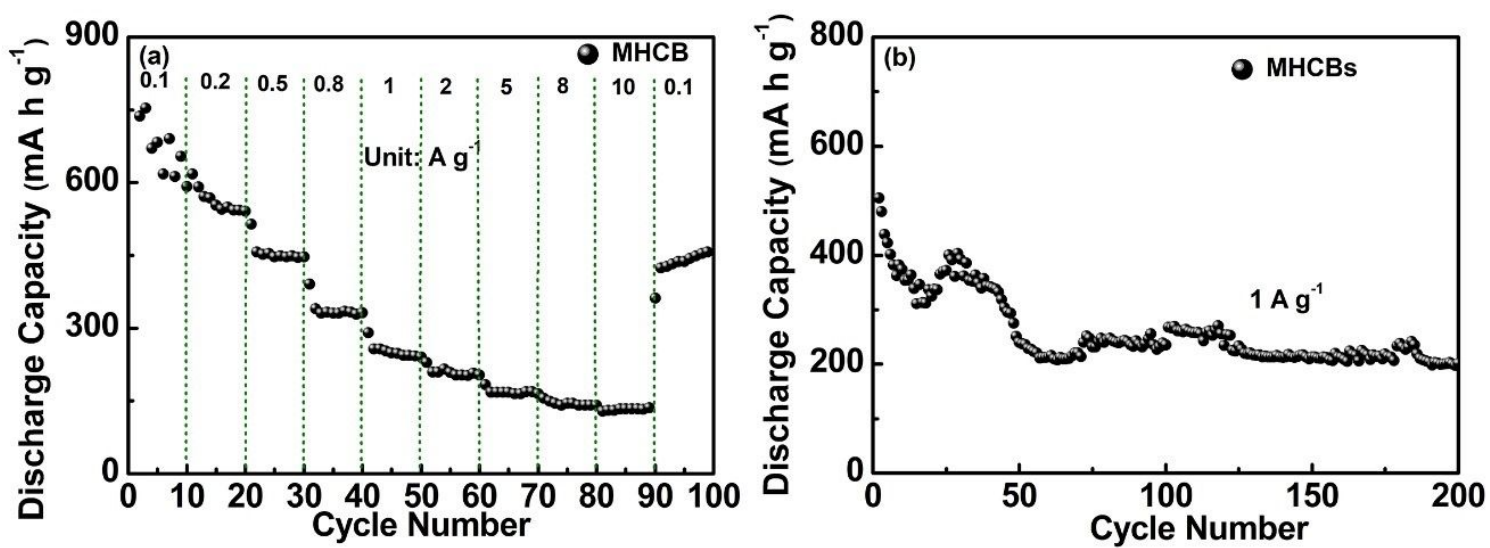

Figure S8. (a) Cycling performance at various current densities ( 0.1 to $\left.10 \mathrm{~A} \mathrm{~g}^{-1}\right)$, (b) Cycling performance of MHCBs and Coulombic efficiency at $1 \mathrm{~A} \mathrm{~g}^{-1}$. 
(a)

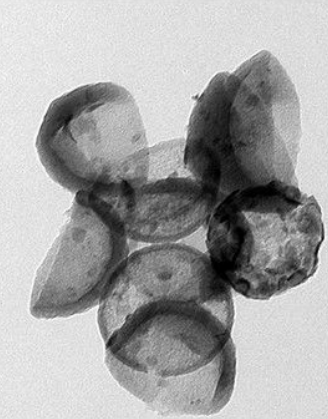

$200 \mathrm{~nm}$ (b) $100 \mathrm{~nm}$ (c)

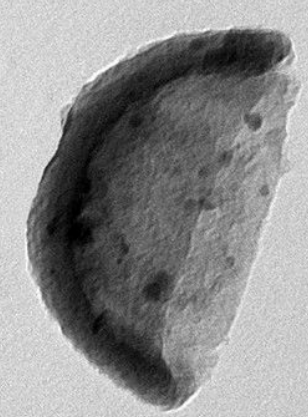

$\underline{50 \mathrm{~nm}}$

Figure S9. (a-c) TEM images at different magnification of the bowl-like $\mathrm{C} @ \mathrm{MoS}_{2}$ after 1000 galvanic charge-discharge cycles at $1 \mathrm{~A} \mathrm{~g}^{-1}$.

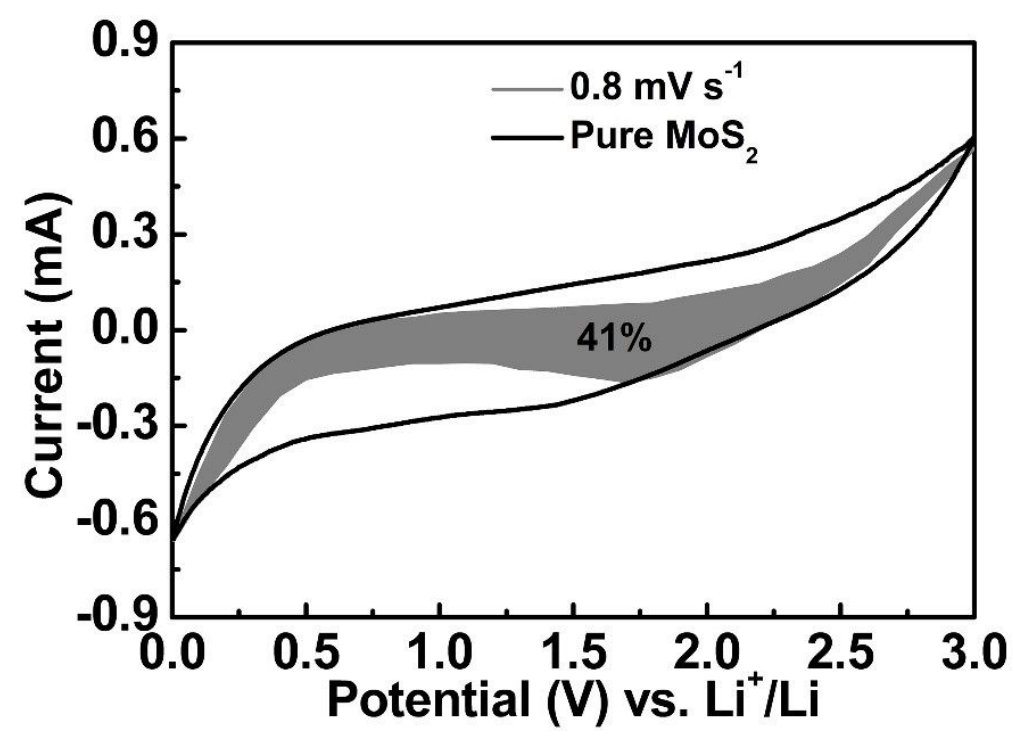

Figure S10. Capacitive contribution at $0.8 \mathrm{mV} \mathrm{s}^{-1}$ of pure $\mathrm{MoS}_{2}$. 
Table S1. Summary of electrochemical performances of different $\mathrm{MoS}_{2}$-based lithium ion anode.

\begin{tabular}{|c|c|c|c|c|}
\hline Sample & $\begin{array}{c}\text { Current density } \\
\qquad\left[\mathrm{A} \mathrm{g}^{-1}\right]\end{array}$ & Cycles & $\begin{array}{l}\text { Reversible capacity } \\
{\left[\mathrm{mA} \mathrm{h} \mathrm{g^{-1 } ]}\right.}\end{array}$ & Reference \\
\hline $\begin{array}{l}\text { bowl-like } \\
\mathrm{C} @ \mathrm{MoS}_{2}\end{array}$ & $0.1 / 1$ & $100 / 1000$ & $798 / 526$ & This work \\
\hline BMCNs@MoS & 0.5 & 400 & 671 & 1 \\
\hline $\mathrm{MoS}_{2} / \mathrm{Mo}_{2} \mathrm{TiC}_{2} \mathrm{~T}_{\mathrm{x}}$ & 0.1 & 100 & 580 & 2 \\
\hline $\mathrm{TiO}_{2} @ \mathrm{NC} @ \mathrm{MoS}_{2}$ & 0.1 & 200 & 590 & 3 \\
\hline $\begin{array}{l}\text { Hierarchical } \\
\mathrm{MoS}_{2}-\mathrm{C} \\
\text { microspheres }\end{array}$ & 1 & 100 & 780 & 4 \\
\hline $\begin{array}{c}\mathrm{C} @ \mathrm{MoS}_{2} \\
\text { microspheres }\end{array}$ & 0.1 & 100 & 652 & 5 \\
\hline $\mathrm{MoS}_{2} / \mathrm{C}$ & 1 & 2500 & 390 & 6 \\
\hline $\begin{array}{l}\mathrm{MoS}_{2} / \mathrm{CFs} \\
\text { hybrids }\end{array}$ & 1.6 & 400 & 630 & 7 \\
\hline $\begin{array}{l}\mathrm{MoS}_{2} \text { yolk-shell } \\
\text { powders }\end{array}$ & 1 & 100 & 362 & 8 \\
\hline PCNF@MoS 2 & 0.5 & 200 & 1304 & 9 \\
\hline $\begin{array}{c}\text { micron-sized } \\
\text { encapsulated-type } \\
\mathrm{MoS}_{2} / \mathrm{C} \\
\text { hybrid structure } \\
\end{array}$ & 1 & 480 & 1250 & 10 \\
\hline $\begin{array}{c}\mathrm{MoS}_{2} @ \mathrm{C} \\
\text { yolk-shell } \\
\text { microspheres }\end{array}$ & 0.5 & 150 & 781 & 11 \\
\hline $\mathrm{NG}-\mathrm{MoS}_{2}$ & 0.1 & 200 & 1205 & 12 \\
\hline
\end{tabular}

Table S2. $R_{\mathrm{ct}}, R_{\mathrm{s}}, \sigma$ and $D_{\mathrm{Li}}$ of pure $\mathrm{MoS}_{2}$ and bowl-like $\mathrm{C} @ \mathrm{MoS}_{2}$

\begin{tabular}{ccccc} 
Samples & $\boldsymbol{R}_{\mathrm{ct}}[\boldsymbol{\Omega}]$ & $\boldsymbol{R}_{\mathrm{S}}[\boldsymbol{\Omega}]$ & $\boldsymbol{\sigma}$ & $\boldsymbol{D}_{\mathrm{Li}}\left[\mathbf{c m}^{\mathbf{2}} \mathbf{s}^{-1}\right]$ \\
\hline Pure $\mathrm{MoS}_{2}$ & 122 & 2.41 & 82.9 & $5.0 \times 10^{-15}$ \\
\hline $\begin{array}{l}\text { Bowl-like } \\
\mathrm{C} @ \mathrm{MoS}_{2}\end{array}$ & 59 & 1.76 & 21.6 & $7.4 \times 10^{-14}$ \\
\hline
\end{tabular}




\section{Reference}

1. Qian, X.; Zhu, G.; Wang, K.; Zhang, F.; Liang, K.; Luo, W.; Yang, J., Bowl-like mesoporous polymer-induced interface growth of molybdenum disulfide for stable lithium storage. Chem. Eng. J. 2020, 381, 122651, DOI 10.1016/j.cej.2019.122651.

2. Chen, C.; Xie, X.; Anasori, B.; Sarycheva, A.; Makaryan, T.; Zhao, M.; Urbankowski, P.; Miao, L.; Jiang, J.; Gogotsi, Y., MoS 2 -on-MXene Heterostructures as Highly Reversible Anode Materials for Lithium-Ion Batteries. Angew. Chem. Int. Ed. 2018, 57 (7), 1846-1850, DOI 10.1016/j.cej.2019.122651.

3. Wang, S.; Guan, B. Y.; Yu, L.; Lou, X. W. D., Rational Design of Three-Layered $\mathrm{TiO}_{2}$ @Carbon@ $\mathrm{MoS}_{2}$ Hierarchical Nanotubes for Enhanced Lithium Storage. Adv. Mater. 2017, 29 (37) 1702724, DOI 10.1002/adma.201702724.

4. Chen, G.; Wang, S.; Yi, R.; Tan, L.; Li, H.; Zhou, M.; Yan, L.; Jiang, Y.; Tan, S.; Wang, D.; Deng, S.; Meng, X.; Luo, H., Facile synthesis of hierarchical $\mathrm{MoS}_{2}-$ carbon microspheres as a robust anode for lithium ion batteries. J. Mater. Chem. A 2016, 4 (24), 9653-9660, DOI 10.1039/c6ta03310e.

5. Wan, Z.; Shao, J.; Yun, J.; Zheng, H.; Gao, T.; Shen, M.; Qu, Q.; Zheng, H., Core-shell structure of hierarchical quasi-hollow $\mathrm{MoS}_{2}$ microspheres encapsulated porous carbon as stable anode for Li-ion batteries. Small 2014, 10 (23), 4975-4981, DOI 10.1002/smll.201401286.

6. Lu, Y.; Zhao, Q.; Zhang, N.; Lei, K.; Li, F.; Chen, J., Facile Spraying Synthesis and High-Performance Sodium Storage of Mesoporous $\mathrm{MoS}_{2} / \mathrm{C}$ Microspheres. Adv. Funct. Mater. 2016, 26 (6), 911-918, DOI 10.1002/adfm.201504062.

7. Ren, D.; Hu, Y.; Jiang, H.; Deng, Z.; Petr, S.; Jiang, H.; Li, C., Salt-Templating Protocol 
To Realize Few-Layered Ultrasmall $\mathrm{MoS}_{2}$ Nanosheets Inlayed into Carbon Frameworks for Superior Lithium-Ion Batteries. ACS Sustain. Chem. Eng. 2016, 4 (3), 1148-1153, DOI 10.1021/acssuschemeng.5b01218.

8. Ko, Y. N.; Kang, Y. C.; Park, S. B., Superior electrochemical properties of $\mathrm{MoS}_{2}$ powders with a MoS $2 @$ void@ $\mathrm{MoS}_{2}$ configuration. Nanoscale 2014, 6 (9), 4508-4512, DOI 10.1039/c4nr00064a.

9. Zhang, C.-L.; Jiang, Z.-H.; Lu, B.-R.; Liu, J.-T.; Cao, F.-H.; Li, H.; Yu, Z.-L.; Yu, S.-H., $\mathrm{MoS}_{2}$ nanoplates assembled on electrospun polyacrylonitrile-metal organic framework-derived carbon fibers for lithium storage. Nano Energy 2019, 61, 104-110, DOI 10.1016/j.nanoen.2019.04.045.

10. Sun, B.; Liu, Q.; Chen, W.; Wang, N.; Gu, J.; Zhang, W.; Su, H.; Zhang, D., Micron-sized encapsulated-type $\mathrm{MoS}_{2} / \mathrm{C}$ hybrid particulates with an effective confinement effect for improving the cycling performance of LIB anodes. J. Mater. Chem. A 2018, 6 (15), 6289-6298, DOI 10.1039/c8ta00674a.

11. Pan, Y.; Zhang, J.; Lu, H., Uniform Yolk-Shell MoS $@$ @Carbon Microsphere Anodes for High-Performance Lithium-Ion Batteries. Chem. Eur. J. 2017, 23 (41), 9937-9945, DOI 10.1002/chem.201701691.

12. Shan, T. T.; Xin, S.; You, Y.; Cong, H. P.; Yu, S. H.; Manthiram, A., Combining Nitrogen-Doped Graphene Sheets and $\mathrm{MoS}_{2}$ : A Unique Film-Foam-Film Structure for Enhanced Lithium Storage. Angew. Chem. Int. Ed. 2016, 55 (41), 12783-12788, DOI 10.1002/anie. 201606870 . 